\title{
Collaborative knowledge management system strategic planning (CKMS2P): a systematic literature review
}

\begin{abstract}
This paper is aimed to identify and review the previous studies pertaining to CKMS2P via a systematic and rigorous approach. The objective is to investigate the strategic plan or guideline on how to develop and implement an effective collaborative knowledge management system (KMS) in order to ease the decision making process. Systematic Literature Review (SLR) method is used to produce a strategic searching method to select, extract, review and discuss relevant studies concerning the research subject by adapting the guidelines established by [11]. A total of 12 previous studies carried out from 2010 till 2014 have been selected to fulfil this objective through thorough selection. Finding shows that there is a very limited study focusing on the strategic implementation of a KMS to enhance the quality of knowledge services in a collaborative environment. Although there is an increase in the studies that recommended KMS guidelines or strategic planning, the proposed solution were mostly unverified or validated. Therefore, it has become a motivation to conduct a study to propose a Collaborative KMS Strategic Planning Model that can significantly enhance the quality of knowledge services in an organization.
\end{abstract}

Keyword: Collaborative knowledge management system strategic planning; Knowledge management; Knowledge management system; Knowledge management strategy 